\title{
Monoclonal Gammopathy of Undetermined Significance and Smoldering Multiple Myeloma
}

\author{
Robert A. Kyle and S. Vincent Rajkumar \\ Department of Medicine, Laboratory Medicine \& Pathology, College of Medicine Mayo Clinic, \\ Stabile 6-28, 200 First Street SW, Rochester, MN 55905, USA
}

Robert A. Kyle: Kyle.robert@mayo.edu

\begin{abstract}
Monoclonal gammopathy of undetermined significance (MGUS) is characterized by the presence of a serum monoclonal (M) protein level less than $3 \mathrm{~g} / \mathrm{dL}$, less than $10 \%$ clonal plasma cells in the bone marrow, and the absence of hypercalcemia, renal insufficiency, anemia, or bone lesions attributable to a clonal plasma cell disorder. Patients may be tested for a monoclonal gammopathy by serum protein electrophoresis, immunofixation, and the free light chain (FLC) assay. The prevalence of MGUS is 3\% for persons more than 50 years of age and 5\% in those more than 70 years of age. The risk of progression to multiple myeloma or a related disorder is $1 \%$ per year. The size and type of M protein, the number of bone marrow plasma cells, and the results of the FLC ratio are independent risk factors for progression. Smoldering multiple myeloma (SMM) is a more advanced premalignant phase than MGUS and is characterized by more than $3 \mathrm{~g} / \mathrm{dL}$ of serum M protein, more than $10 \%$ clonal plasma cells in the bone marrow, or both, with no evidence of endorgan damage.
\end{abstract}

\section{Keywords}

Plasma cell disorders

\section{Introduction}

The plasma cell disorders are characterized by the proliferation of monoclonal plasma cells. These disorders include multiple myeloma; Waldenström's macroglobulinemia (WM); immunoglobulin light chain (AL) amyloidosis; solitary plasmacytoma of bone; solitary extramedullary plasmacytoma; POEMS syndrome (polyneuropathy, organomegaly, endocrinopathy, M protein, skin changes); plasma cell leukemia; nonsecretory myeloma; $\gamma$, $a$, and $\mu$ heavy-chain diseases; monoclonal gammopathy of undetermined significance (MGUS); and smoldering multiple myeloma (SMM). MGUS and SMM are characterized by the absence of end-organ damage (CRAB-hypercalcemia, renal insufficiency, anemia, bone lesions).

\section{Monoclonal Gammopathy of Undetermined Significance}

The term "monoclonal gammopathy of undetermined significance" was introduced over three decades ago. The disorder was defined as having a serum monoclonal (M) protein less

C) Springer Science+Business Media, LLC 2010

Correspondence to: Robert A. Kyle, Kyle.robert@mayo. edu.

Disclosure No potential conflicts of interest relevant to this article were reported. 
than $3.0 \mathrm{~g} / \mathrm{dL}$; less than $10 \%$ clonal plasma cells in the bone marrow; little or no M protein in the urine; and no lytic bone lesions, renal insufficiency, hypercalcemia, or anemia related to the plasma cell proliferative process [1].

\section{Recognition of Monoclonal Proteins}

Agarose gel electrophoresis is the preferred method for detection. If a localized band or spike is found, immunofixation must be performed to confirm the presence of an M protein and to determine its immunoglobulin heavy chain class and its light chain type.

A patient who presents with nonspecific backache, mild anemia, hypercalcemia, osteopenia, osteolytic lesions, or mild renal insufficiency can be screened for the presence of an M protein using serum protein electrophoresis, immunofixation, and the free light chain (FLC) assay. In a series of 428 patients with multiple myeloma, AL amyloidosis, MGUS, SMM, or solitary plasmacytoma, only two patients were missed with these three screening tests. Electrophoresis and immunofixation of an aliquot from a 24-hour urine specimen was not necessary for screening [2]. In a recent report of 1,877 patients with a monoclonal plasma cell proliferative disorder, $94 \%$ of patients were detected with serum protein electrophoresis and the FLC assay. Serum protein electrophoresis, immunofixation, FLC assay, and electrophoresis plus immunofixation of a 24-hour urine aliquot recognized $98.6 \%$ of patients. Just two tests, serum protein electrophoresis and the serum FLC assay, detected $100 \%$ of patients with multiple myeloma or macroglobulinemia, $99.5 \%$ of those with SMM, $96 \%$ of patients with AL amyloidosis, and $89 \%$ of patients with MGUS. Thus, most patients were found by screening with just two tests [3•].

An M protein should be sought in any patient in whom there is even a low suspicion of multiple myeloma, WM, AL amyloidosis, or related disorders.

\section{Prevalence of MGUS}

In Sweden, the United States, and western France, approximately $1.5 \%$ of persons older than 50 years of age and 3\% of the population more than 70 years of age have an $\mathrm{M}$ protein without evidence of multiple myeloma or a related disorder [4-6]. The frequency of MGUS increases as the patient ages (Table 1) [7]. Of 111 patients older than 80 years, 10\% had an M protein in one US study [8].

The prevalence of MGUS is higher in African Americans than in Caucasians. For example, $8.6 \%$ of 916 black patients had an M protein, compared with $3.6 \%$ of white patients in North Carolina [9]. In a study of 4 million African American and white male veterans admitted to Veterans Affairs Hospitals, the prevalence of MGUS in African Americans was 3.0-fold higher than in Caucasians [10]. The increased prevalence in blacks was confirmed by a study of 917 men 50 to 74 years of age from Ghana. The age-adjusted prevalence was 5.8\%, which is almost twice as high as in Caucasians. However, the prevalence did not increase with advancing age [11]. On the other hand, prevalence of MGUS has been reported to be lower in Japanese patients. The prevalence of MGUS in Nagasaki City, Japan, was $2.4 \%$ in patients 50 years of age or older and rose from $1.0 \%$ among persons 42 to 49 years of age to $4.4 \%$ among those 80 years or older [12].

In a population-based study, serum samples were obtained from 21,463 (77\%) of the 28,038 enumerated residents of Olmsted County, Minnesota, who were 50 years of age or older. MGUS was found in 694 (3.2\%) of the population. The age-adjusted rates were higher in men than in women $-4.0 \%$ versus $2.7 \%(P<0.001)$. The prevalence was $7.5 \%$ among those 85 years of age or older and $5.3 \%$ in patients 70 years or older. The amount of M protein was less than $1.0 \mathrm{~g} / \mathrm{dL}$ in $63.5 \%$ and $2 \mathrm{~g} / \mathrm{dL}$ or more in only $4.5 \%$ of the 694 patients. 
Reduced concentration of uninvolved immunoglobulins was found in $28 \%$ of 447 persons tested [7].

\section{Etiology of MGUS}

MGUS is more common in first-degree relatives of both MGUS and multiple myeloma patients. One study assessed 911 relatives of 97 MGUS and 232 multiple myeloma probands. The prevalence of MGUS in first-degree relatives of the MGUS and multiple myeloma probands was $1.9 \%$ at ages 40 to 49 years, $6.9 \%$ in those 50 to 59 years of age, $11.6 \%$ in persons 60 to 69 years of age, $14.6 \%$ in those 70 to 79 years old, and $21 \%$ in those 80 years of age or older. The relative risk of MGUS in relatives of multiple myeloma patients was increased twofold, whereas the risk in relatives of MGUS patients was increased 3.3-fold. The increased risk of MGUS in first-degree relatives of patients with MGUS or multiple myeloma suggests a shared environmental and/or genetic effect [13•].

Increased radiation exposure may play a role. Among 52,525 Nagasaki atomic bomb survivors were 1,082 MGUS patients. The risk of MGUS was increased 1.4-fold in people within $1.5 \mathrm{~km}$ of the explosion (2.7\% prevalence) compared with those beyond $3.0 \mathrm{~km}$ (2.0\% prevalence). Those exposed when younger than 20 years had an increased prevalence of MGUS, but there was no difference among those who were exposed when older than 20 years of age [14•].

Multiple case control studies have suggested an elevated risk of multiple myeloma among agricultural workers [15•]. Insecticides, herbicides, and fungicides have all been hypothesized as the basis for this increased risk. In a study of 555 men from a wellcharacterized prospective cohort of those applying restricted-use pesticides, $6.8 \%$ of those older than 50 years had MGUS, compared with $3.7 \%$ in 9,469 men from Olmsted County, Minnesota. The age-adjusted prevalence of MGUS was 1.9-fold higher among the male pesticide workers. There was a 5.6-fold increased risk among the users of dieldrin, a 3.9-fold risk for those using a carbon tetrachloride-carbon disulfide fumigant mixture, and a 2.4-fold increased risk of MGUS prevalence for those using the fungicide chlorothalonil. In short, the prevalence of MGUS among workers applying pesticides was about twice that in a population-based sample of men from Minnesota. This finding strongly suggests that pesticide exposure is a risk factor for MGUS [16•].

\section{Long-Term Outcome}

MGUS is a common finding in medical practice. Determining whether the monoclonal protein will remain stable or progress to multiple myeloma or a related disorder is of vital importance.

Mayo Clinic Referral Population-In one study, we reviewed the medical records of all patients with monoclonal gammopathy who were seen at Mayo Clinic from 1956 to 1970. Those with multiple myeloma, WM, AL amyloidosis, lymphoma, or related disorders were excluded. A total of 241 patients with MGUS were observed for 3,579 person-years (median, 13.7 years; range, 0-39 years). Of greatest interest were the 64 patients who developed multiple myeloma, WM, AL amyloidosis, or a lymphoproliferative disorder. These patients were observed for a median of 10.4 years (range, 1-32 years) before diagnosis of a serious lymphoplasma cell proliferative disorder. The actuarial rate of disease progression was $17 \%$ at 10 years, $34 \%$ at 20 years, and $39 \%$ at 25 years, a progression rate of approximately $1.5 \%$ per year. Of these 64 patients, 44 (69\%) developed multiple myeloma. The diagnosis of multiple myeloma occurred from 1 to 32 years (median, 10.6 years) after the recognition of MGUS. 
Southeastern Minnesota Population-Based Study-In an effort to confirm the findings of the 241 Mayo Clinic patients (who came from the entire United States and several foreign countries), we reported on a separate study of 1,384 patients with MGUS from the 11 counties of southeastern Minnesota who were evaluated at Mayo Clinic from 1960 to 1994 [17]. The median age at recognition of MGUS was 72 years, which is 8 years older than the median age for the 241 patients in the cohort from all parts of the world. Of the 1,384 Minnesota patients, who were observed for a total of 11,009 person-years (median, 15.4 years; range, $0-35$ years), 963 (70\%) died. During follow-up, multiple myeloma, AL amyloidosis, lymphoma with IgM serum protein, WM, plasmacytoma, or chronic lymphocytic leukemia (CLL) developed in 115 patients (8\%). The cumulative probability of progression to one of these disorders was approximately $1 \%$ per year. The rate of progression was $10 \%$ at 10 years, $21 \%$ at 20 years, and $26 \%$ at 25 years. It must be emphasized that patients are at risk for progression of MGUS even after more than 25 years' observation.

The number of patients with progression to a plasma cell disorder $(n=115)$ was 7.3 times the number expected on the basis of the incidence rates for these conditions in the general population.

Other Series-Several other series have reported similar findings. Of the 64 patients with MGUS in a Swedish study, 11\% had some evidence of progression during 20 years of follow-up [18]. In another group, 13 of 128 patients with MGUS developed a malignant plasma cell disease during the median follow-up of 66 months [19]. In another study, 6.8\% of 335 persons with MGUS progressed during a median follow-up of 70 months [20]. In a cohort of 1,324 patients with MGUS in North Jutland, Denmark, malignant transformation caused death in 97 patients, compared with 4.9 deaths expected [21]. In the Danish Cancer Registry, 64 new cases of malignancy (5 expected; relative risk (RR), 12.9) were found among 1,229 patients with MGUS [22]. In a series of 504 patients with MGUS from Iceland, a related malignancy developed in $51(10 \%)$ after a median follow-up of 6 years [23].

\section{Causes of Progression}

The cause of malignant progression of MGUS is not well understood. Genetic changes, bone marrow angiogenesis, and cytokines related to myeloma bone disease may all play a role in the progression of MGUS to multiple myeloma or a related disorder.

Genetic Changes-Of patients with multiple myeloma, 60\% have an immunoglobulin $\mathrm{H}$ (IgH) (14q32) translocation with fluorescence in situ hybridization (FISH) [24]. In one series, IgH translocations were found in 27 (46\%) of 59 patients with MGUS [25]. In another report, translocations consisting of $\mathrm{t}(11 ; 14)(\mathrm{q} 13 ; \mathrm{q} 32)$ were found in $25 \%, \mathrm{t}(4 ; 14)$ (p16.3;q32) in 9\%, and $\mathrm{t}(14 ; 16)(\mathrm{q} 32 ; \mathrm{q} 23)$ in 5\% [26]. These translocations lead to the dysregulation of oncogenes such as cyclin D, c-MAF, FGFR3-MMSET, and cyclin D3; they may play a role in the initiation of the MGUS clone rather than in the progression of MGUS to multiple myeloma. In another report, Chng et al. [27] reported that $40 \%$ of 28 patients with SMM or MGUS had hyperdiploidy. Deletions of chromosome 13 are found in similar frequencies in both multiple myeloma and MGUS. Aberrant methylation of the 5' gene promoter regions of tumor suppressor genes have been observed in MGUS.

Gene expression profiling with microarray data showed that Myc upregulation and increasing chromosomal instability characterized the evolution from normal plasma cells to relapsed multiple myeloma. Some patients with MGUS shared biologic features with relapsed myeloma, which may identify a high-risk MGUS signature [28•]. 
Bone Marrow Angiogenesis-Bone marrow angiogenesis is increased in multiple myeloma and is of prognostic value. We reported that the median microvessel density was 1.3 in normal controls, 1.7 in AL amyloidosis, 3 in MGUS, 4 in SMM, 11 in multiple myeloma, and 20 in relapsed multiple myeloma [29]. The increase in angiogenesis that occurs with progression appears to alter the balance between proangiogenic and antiangiogenic effects.

Bone Disease-The occurrence of lytic bone lesions is the most important feature that differentiates multiple myeloma from MGUS. In a study of 488 Olmsted County residents with MGUS, we found a 2.7-fold increase in axial fractures but no increase in limb fractures [30]. Bone lesions are caused by osteoclast activation and inhibition of osteoblast differentiation. Overexpression of various cytokines such as receptor activator of nuclear factor kappa B ligand (RANKL) and macrophage inhibitory protein 1 a (MIP1a) play an important role in the development of bone lesions. Osteoprotegerin (OPG) modulates RANKL. The excess RANKL or reduced levels of OPG may produce myeloma bone disease. Interleukin- $1 \beta$ also has strong osteoclast-activating factor activity. Interleukin-6 (IL-6) and tumor necrosis factor-a may also play a role.

\section{Predictors of Progression}

It is not possible to distinguish a patient with MGUS who will remain stable from one in whom progression to a plasma cell malignancy will develop. A number of parameters are helpful in predicting the likelihood of progression of MGUS to multiple myeloma.

Concentration of Serum M Protein-In the series of 1,384 MGUS patients from southeastern Minnesota, the most important predictor of progression to a plasma cell disorder was the concentration of the $\mathrm{M}$ protein at diagnosis. If the initial $\mathrm{M}$ protein value was less than $0.5 \mathrm{~g} / \mathrm{dL}$, the risk of progression at 20 years was $14 \%$, whereas those presenting with an M protein of $2.5 \mathrm{~g} / \mathrm{dL}$ had a $49 \%$ risk of progression [17].

Type of Serum M Protein-In our series of 1,384 patients, patients with an IgM or an IgA monoclonal protein had an increased risk of progression, compared with patients who had an IgG protein [17].

Bone Marrow Plasma Cells-Baldini et al. [20] reported a transformation rate of 6.8\% when the bone marrow plasma cell level was less than 10\%. Cesana et al. [31] found that having more than $5 \%$ bone marrow plasma cells was an independent risk factor for progression.

Abnormal Serum FLC Ratio-In a study of 1,148 of the 1,384 MGUS patients from southeastern Minnesota, we found an abnormal FLC ratio in 33\%. At a median follow-up of 15 years, progression occurred in $87(7.6 \%)$ of these patients. The risk of progression in patients with an abnormal FLC ratio was higher than in patients with a normal ratio (hazard ratio (HR), 3.5) and was independent of the concentration and type of serum M protein [32].

\section{Risk Stratification Model for MGUS}

We have developed a risk stratification model for progression of MGUS that predicts the risk of progression using simple laboratory markers. Patients with risk factors consisting of an elevated serum M protein value ( $\geq 1.5 \mathrm{~g} / \mathrm{dL}$ ), a non-IgG MGUS, and an abnormal serum FLC ratio had a risk of progression at 20 years of 58\% (high risk), compared with only 5\% when none of these risk factors were present (low risk) [32]. After 20 years of follow-up of our southeastern Minnesota patients, plasma cell disorders had developed in 10\%, whereas $72 \%$ had died of other causes. 


\section{Management of MGUS}

Patients with MGUS should be tested again in 4 to 6 months to exclude an evolving multiple myeloma (MM). Patients with low-risk MGUS may be reevaluated every 2 years, whereas those with high-risk MGUS should be followed annually for life or until they develop an unrelated condition that severely limits life expectancy. It is important to detect a serious plasma cell disorder before complications such as renal failure or pathologic fractures occur.

At the time of the follow-up examination, a careful history and physical examination should be performed, looking for symptoms or signs of one of the malignant disorders known to evolve from MGUS. The serum and urine M protein values should be measured, as well as the complete blood count, calcium, and creatinine. Patients should always be told to obtain medical evaluation promptly if clinical symptoms occur.

\section{Association of MGUS with Other Diseases}

The association of two diseases depends on the frequency with which each occurs independently. Furthermore, an apparent association may occur because of referral practices or other selection of patient groups. These apparent associations must be evaluated by appropriate epidemiologic and statistical methods.

We have studied the association of MGUS with all diseases in a population-based cohort from Olmsted County, Minnesota. Among the 17,398 patients tested, 605 cases of MGUS and 16,793 negative controls were identified. The computerized Mayo Medical Index was used to obtain information on all diagnoses between 1975 and 2006, for a total of 422,663 person-years of observation. Seventy-five diagnoses for which a potential association with MGUS has been reported were studied. We were able to confirm a significant disease association in 14 diagnoses. Of these 14 diagnoses, five are known to evolve from MGUS: multiple myeloma, AL amyloidosis, lymphoproliferative disorders, WM, and other unclassified plasma cell proliferative disorders. Hip and vertebral fractures, osteoporosis, and hypercalcemia were all significantly increased with MGUS, even in the absence of progression to multiple myeloma. We also confirmed the association of MGUS with chronic inflammatory demyelinating neuropathy. We found no significant association of MGUS with the 61 remaining disease diagnoses, which suggests that most of the previously reported associations are either coincidental or clinically insignificant [33••].

The associations of MGUS with lymphoproliferative disorders, leukemia, other hematologic diseases, connective tissue disorders, neurologic diseases, dermatologic problems, endocrine disorders, liver disease, immunosuppression, and many miscellaneous conditions have previously been reviewed [34]. We identified 71 patients in a cohort of 77,469 healthy adults enrolled in a nationwide population-based prospective prostate, lung, colorectal, and ovarian (PLCO) cancer screening trial. During the course of the study, serially collected serum samples were obtained. We found that MGUS was present in $100 \%$ of patients 2 years before the recognition of multiple myeloma. MGUS was recognizable in $95 \%$ of patients 5 years prior to the diagnosis of multiple myeloma, and 82.4\% had MGUS more than 8 years before the recognition of multiple myeloma [35•]. Weiss et al. [36] found an M protein in the serum of 27 of 30 patients prior to the diagnosis of multiple myeloma.

\section{Smoldering (Asymptomatic) Multiple Myeloma}

SMM is an asymptomatic plasma cell proliferative disorder characterized by the presence of a serum $\mathrm{M}$ protein level $3 \mathrm{~g} / \mathrm{dL}$ or greater, $10 \%$ or more clonal plasma cells in the bone marrow, or both. It represents a more advanced premalignant stage than MGUS. Small amounts of $\mathrm{M}$ protein in the urine and a reduction of uninvolved immunoglobulins in the serum are not uncommon in SMM. Most importantly, as in MGUS, there is no evidence of 
end-organ damage-no hypercalcemia, renal insufficiency, anemia, or bone lesions. In fact, SMM patients have MGUS from the biologic standpoint, but the diagnostic criteria differ from the MGUS criteria to highlight the higher risk of progression in SMM compared with MGUS. It is also important to recognize this entity because patients with SMM should not be treated until symptomatic disease occurs.

SMM accounts for approximately $10 \%$ to $15 \%$ of all cases of newly diagnosed multiple myeloma. The prevalence is difficult to determine because some reports include asymptomatic patients with lytic lesions on the skeletal survey. Others exclude patients with bone lesions on the skeletal radiographs but include patients who have lytic lesions detected by MRI.

\section{Risk of Progression}

Many patients with SMM progress to symptomatic disease, and the risk of progression is higher than in patients presenting with MGUS. We found that the risk of progression was $10 \%$ per year for the first 5 years, 3\% per year for the next 5 years, and then $1 \%$ to $2 \%$ per year for the next 10 years [37••]. The decrease in risk of progression with time in SMM is different from the fixed $1 \%$ per year risk in MGUS. The median duration of progression to symptomatic disease ranges from 1 to 5 years.

\section{Predictors of Progression}

Two subsets of SMM have been described: evolving SMM, which is characterized by a progressive increase of the serum M protein level; and non-evolving SMM, which is characterized by a stable $\mathrm{M}$ protein value that abruptly increases when symptomatic multiple myeloma develops [38].

An increase in the number or proliferation rate of circulating plasma cells determined by immunofluorescence is an important risk factor for progression. In a study of 57 patients, we found that the median time to progression was 9 months for those with abnormal circulating plasma cell values and 30 months for those with normal results [39].

In another report, the three most important prognostic factors for progression of SMM were a serum $\mathrm{M}$ protein level greater than $3.0 \mathrm{~g} / \mathrm{dL}$, an IgA immunoglobulin, and urine $\mathrm{M}$ protein excretion of more than $50 \mathrm{mg}$ per day. Patients with two or three of these risk factors had a median time to progression of 17 months, whereas those with one risk factor had a median time to progression of 40 months, and those with none of the factors had a progression time of 95 months [40]. In this study, patients with abnormal MRI findings had a time to progression of 21 months.

\section{Risk Stratification of SMM}

As with MGUS, we have developed a risk-stratification model for SMM to predict risk of progression. Three risk factors have significant prognostic value: (1) bone marrow plasma cells greater than $10 \%$; (2) serum M protein greater than $3.0 \mathrm{~g} / \mathrm{dL}$; and (3) abnormal FLC ratio less than 0.125 or greater than 8 . The probability of progression at 5 years was $25 \%$ for those with one risk factor, $51 \%$ in the presence of two risk factors, and $76 \%$ if all three risk factors were present [41].

\section{Management of SMM}

If there are doubts about the differentiation of SMM from multiple myeloma and whether to begin chemotherapy immediately, one should withhold treatment and reevaluate in 2 or 3 months. One must keep in mind that patients with SMM may remain stable for a long time. 
Patients should be reevaluated 3 to 6 months after the recognition of SMM; if they are stable, they can be seen at 6-month intervals.

There is no evidence that overall survival is lengthened by giving chemotherapy to asymptomatic patients. In one study, 50 patients with asymptomatic stage 1 multiple myeloma were randomized to observation versus chemotherapy with melphalan and prednisone. No differences were found in overall survival between the two groups. In another report, 44 patients with asymptomatic multiple myeloma had no difference in survival with immediate or deferred therapy.

Clinical trials are ongoing to determine whether the use of agents such as bisphosphonates, interleukin-1 $\beta$ inhibitors, clarithromycin, or dehydroepiandrosterone can delay progression and prolong overall survival. Phase 3 randomized trials are needed to determine the durability of response and effect on overall survival without harm to the patient before treatment can be recommended for patients with SMM.

\section{Conclusions}

MGUS is defined by the presence of a serum M protein concentration less than $3 \mathrm{~g} / \mathrm{dL}$, less than $10 \%$ clonal plasma cells in the bone marrow, and absence of hypercalcemia, renal insufficiency, anemia, or bone lesions attributable to a clonal plasma cell disorder. The prevalence of MGUS is approximately $3 \%$ of the general population older than 50 years of age. Factors that affect the prevalence of MGUS include age, gender, race, exposure to pesticides, and family history. MGUS progresses to multiple myeloma or a related disorder at a rate of $1 \%$ per year. The concentration and type of $\mathrm{M}$ protein, number of bone marrow plasma cells, and the FLC ratio are independent risk factors for progression. SMM represents a more advanced premalignant phase than MGUS, characterized by the presence of $3 \mathrm{gm} / \mathrm{dL}$ or more serum M protein and/or $10 \%$ or more clonal plasma cells in the bone marrow, with no evidence of end-organ damage. No therapy is needed for MGUS or SMM. Patients with low-risk MGUS should be reexamined in 6 months and then, if stable, every 2 years or when symptoms occur. All other patients with MGUS should be checked in 6 months and then yearly thereafter. Patients with SMM need closer follow-up (every 3-4 months initially) and are candidates for clinical trials evaluating strategies to prevent progression.

\section{Acknowledgments}

Supported in part by research grants CA 62242 and CA107476 by the National Institutes of Health, Bethesda, Maryland, USA.

\section{References}

Papers of particular interest, published recently, have been highlighted as:

- $\quad$ Of importance

-• Of major importance

1. Kyle RA. Monoclonal gammopathy of undetermined significance. Natural history in 241 cases. Am J Med. 1978; 64:814-826. [PubMed: 645746]

2. Katzmann JA, Dispenzieri A, Kyle RA, et al. Elimination of the need for urine studies in the screening algorithm for monoclonal gammopathies by using serum immunofixation and free light chain assays. Mayo Clin Proc. 2006; 81:1575-1578. [PubMed: 17165636]

3•. Katzmann JA, Kyle RA, Benson J, et al. Screening panels for detection of monoclonal gammopathies. Clin Chem. 2009; 55:1517. Serum protein electrophoresis and the serum FLC 
assay detected $100 \%$ of patients with multiple myeloma or WM and $99.5 \%$ of those with SMM. [PubMed: 19520758]

4. Axelsson U, Bachmann R, Hallen J. Frequency of pathological proteins (M-components) in 6,995 sera from an adult population. Acta Med Scand. 1966; 179:235-247. [PubMed: 4160039]

5. Kyle RA, Finkelstein S, Elveback LR, Kurland LT. Incidence of monoclonal proteins in a Minnesota community with a cluster of multiple myeloma. Blood. 1972; 40:719-724. [PubMed: 4627911]

6. Saleun JP, Vicariot M, Deroff P, Morin JF. Monoclonal gammopathies in the adult population of Finistere, France. J Clin Pathol. 1982; 35:63-68. [PubMed: 6801095]

7. Kyle RA, Therneau TM, Rajkumar SV, et al. Prevalence of monoclonal gammopathy of undetermined significance. N Engl J Med. 2006; 354:1362-1369. [PubMed: 16571879]

8. Crawford J, Eye MK, Cohen HJ. Evaluation of monoclonal gammopathies in the "well" elderly. Am J Med. 1987; 82:39-45. [PubMed: 3492143]

9. Cohen HJ, Crawford J, Rao MK, et al. Racial differences in the prevalence of monoclonal gammopathy in a community-based sample of the elderly. Am J Med. 1998; 104:439-444. Erratum appears in Am J Med 1998, 105 (4):362. [PubMed: 9626026]

10. Landgren O, Gridley G, Turesson I, et al. Risk of monoclonal gammopathy of undetermined significance (MGUS) and subsequent multiple myeloma among African American and white veterans in the United States. Blood. 2006; 107:904-906. [PubMed: 16210333]

11. Landgren O, Katzmann JA, Hsing AW, et al. Prevalence of monoclonal gammopathy of undetermined significance among men in Ghana. Mayo Clin Proc. 2007; 82:1468-1473. [PubMed: 18053453]

12. Iwanaga M, Tagawa M, Tsukasaki K, et al. Prevalence of monoclonal gammopathy of undetermined significance: study of 52,802 persons in Nagasaki City, Japan. Mayo Clin Proc. 2007; 82:1474-1479. [PubMed: 18053454]

13•. Vachon CM, Kyle RA, Therneau TM, et al. Increased risk of monoclonal gammopathy in firstdegree relatives of patients with multiple myeloma or monoclonal gammopathy of undetermined significance. Blood. 2009; 114:785-790. The relative risk of MGUS in relatives of multiple myeloma patients was increased twofold, whereas the risk in relatives of MGUS patients was increased 3.3-fold. This suggests a shared environmental or genetic defect. [PubMed: 19179466]

14 . Iwanaga M, Tagawa M, Tsukasaki K, et al. Relationship between monoclonal gammopathy of undetermined significance and radiation exposure in Nagasaki atomic bomb survivors. Blood. 2009; 113:1639-1650. This is the first paper describing the relationship between MGUS and radiation exposure in the Nagasaki atomic bomb explosion. It indicates that only those who were exposed when less than 20 years of age or those who were within $1.5 \mathrm{~km}$ of the explosion had an increased risk of MGUS. [PubMed: 18849487]

15•. Kyle RA, Rajkumar SV. Epidemiology of the plasma-cell disorders. Best Pract Res Clin Haematol. 2007; 20:637-64. This is a comprehensive review of the epidemiology of multiple myeloma and related disorders. [PubMed: 18070711]

16•. Landgren O, Kyle RA, Hoppin JA, et al. Pesticide exposure and risk of monoclonal gammopathy of undetermined significance in the Agricultural Health Study. Blood. 2009; 113:6386-6391. Agricultural workers who are exposed to insecticides, herbicides, and fungicides have a 1.9-fold higher risk of MGUS than a normal population. [PubMed: 19387005]

17. Kyle RA, Therneau TM, Rajkumar SV, et al. A long-term study of prognosis in monoclonal gammopathy of undetermined significance. N Engl J Med. 2002; 346:564-569. [PubMed: 11856795]

18. Axelsson U. A 20-year follow-up study of 64 subjects with M-components. Acta Med Scand. 1986; 219:519-522. [PubMed: 3739756]

19. Blade J, Lopez-Guillermo A, Rozman C, et al. Malignant transformation and life expectancy in monoclonal gammopathy of undetermined significance. Br J Haematol. 1992; 81:391-394.

[PubMed: 1390212]

20. Baldini L, Guffanti A, Cesana BM, et al. Role of different hematologic variables in defining the risk of malignant transformation in monoclonal gammopathy. Blood. 1996; 87:912-918.

[PubMed: 8562962] 
21. Gregersen H, Ibsen J, Mellemkjoer L, et al. Mortality and causes of death in patients with monoclonal gammopathy of undetermined significance. Br J Haematol. 2001; 112:353-357. [PubMed: 11167828]

22. Gregersen H, Mellemkjaer L, Salling Ibsen J, et al. Cancer risk in patients with monoclonal gammopathy of undetermined significance. Am J Hematol. 2000; 63:1-6. [PubMed: 10602159]

23. Ogmundsdóttir HM, Haraldsdóttir V, M Jóhannesson G, et al. Monoclonal gammopathy in Iceland: a population-based registry and follow-up. Br J Haematol. 2002; 118:166-173. [PubMed: 12100144]

24. Avet-Loiseau H, Li JY, Facon T, et al. High incidence of translocations t(11;14)(q13;q32) and $\mathrm{t}(4 ; 14)(\mathrm{p} 16 ; \mathrm{q} 32)$ in patients with plasma cell malignancies. Cancer Res. 1998; 58:5640-5645. [PubMed: 9865713]

25. Avet-Loiseau H, Facon T, Daviet A, et al. 14q32 translocations and monosomy 13 observed in monoclonal gammopathy of undetermined significance delineate a multistep process for the oncogenesis of multiple myeloma. Intergroupe Francophone du Myélome. Cancer Res. 1999; 59:4546-4550. [PubMed: 10493504]

26. Fonseca R, Bailey RJ, Ahmann GJ, et al. Genomic abnormalities in monoclonal gammopathy of undetermined significance. Blood. 2002; 100:1417-1424. [PubMed: 12149226]

27. Chng WJ, Van Wier SA, Ahmann GJ, et al. A validated FISH trisomy index demonstrates the hyperdiploid and nonhyperdiploid dichotomy in MGUS. Blood. 2005; 106:2156-2161. [PubMed: 15920009]

28- Anguiano A, Tuchman SA, Acharya C, et al. Gene expression profiles of tumor biology provide a novel approach to prognosis and may guide the selection of therapeutic targets in multiple myeloma. J Clin Oncol. 2009; 27:4197-4203. This is an excellent article on gene expression profiling, a technique that will be of greater significance in the future. [PubMed: 19636021]

29. Rajkumar SV, Mesa RA, Fonseca R, et al. Bone marrow angiogenesis in 400 patients with monoclonal gammopathy of undetermined significance, multiple myeloma, and primary amyloidosis. Clin Cancer Res. 2002; 8:2210-2216. [PubMed: 12114422]

30. Melton LJ 3rd, Rajkumar SV, Khosla S, et al. Fracture risk in monoclonal gammopathy of undetermined significance. J Bone Miner Res. 2004; 19:25-30. [PubMed: 14753733]

31. Cesana C, Klersy C, Barbarano L, et al. Prognostic factors for malignant transformation in monoclonal gammopathy of undetermined significance and smoldering multiple myeloma. J Clin Oncol. 2002; 20:1625-1634. [PubMed: 11896113]

32. Rajkumar SV, Kyle RA, Therneau TM, et al. Serum free light chain ratio is an independent risk factor for progression in monoclonal gammopathy of undetermined significance. Blood. 2005; 106:812-817. [PubMed: 15855274]

33••. Bida JP, Kyle RA, Therneau TM, et al. Disease associations with monoclonal gammopathy of undetermined significance: a population-based study of 17,398 patients. Mayo Clin Proc. 2009; 84:685-693. This is the first report of disease associations in a large number of patients with MGUS. It serves as a guide for determining the importance of clinical entities associated with MGUS. [PubMed: 19648385]

34. Kyle RA, Rajkumar SV. Monoclonal gammopathy of undetermined significance. Br J Haematol. 2006; 134:573-589. [PubMed: 16938117]

35•. Landgren O, Kyle RA, Pfeiffer RM, et al. Monoclonal gammopathy of undetermined significance (MGUS) consistently precedes multiple myeloma: a prospective study. Blood. 2009; 113:54125417. Virtually all patients with multiple myeloma have a preceding MGUS. [PubMed: 19179464]

36. Weiss BM, Abadie J, Verma P, et al. A monoclonal gammopathy precedes multiple myeloma in most patients. Blood. 2009; 113:5418-5422. [PubMed: 19234139]

37••. Kyle RA, Remstein ED, Therneau TM, et al. Clinical course and prognosis of smoldering (asymptomatic) multiple myeloma. N Engl J Med. 2007; 356:2582-2590. This is a long-term follow-up of a large series of patients with SMM. The risk of progression was $10 \%$ per year for the first 5 years but fell to $1 \%$ to $2 \%$ annually after 10 years of observation. [PubMed: 17582068]

38. Rosinol L, Blade J, Esteve J. Smoldering multiple myeloma: natural history and recognition of an evolving type. Br J Haematol. 2003; 123:631-636. [PubMed: 14616966] 
39. Witzig TE, Kyle RA, O'Fallon WM, Greipp PR. Detection of peripheral blood plasma cells as a predictor of disease course in patients with smouldering multiple myeloma. Br J Haematol. 1994; 87:266-272. [PubMed: 7947266]

40. Weber DM, Dimopoulos MA, Moulopoulos LA, et al. Prognostic features of asymptomatic multiple myeloma. Br J Haematol. 1997; 97:810-814. [PubMed: 9217181]

41. Dispenzieri A, Kyle RA, Katzmann JA, et al. Immunoglobulin free light chain ratio is an independent risk factor for progression of smoldering (asymptomatic) multiple myeloma. Blood. 2008; 111:785-789. [PubMed: 17942755] 


\section{Table 1}

Prevalence of MGUS according to age group and sex among residents of Olmsted County, Minnesota

\begin{tabular}{|c|c|c|c|}
\hline Age, $y$ & Men, $\%^{a}$ & Women, $\%^{a}$ & Total, $\%$ \\
\hline $50-59$ & 2.0 & 1.4 & 1.7 \\
\hline $60-69$ & 3.7 & 2.3 & 3.0 \\
\hline $70-79$ & 5.6 & 3.8 & 4.6 \\
\hline$\geq 80$ & 8.3 & 6.0 & 6.6 \\
\hline Total & $3.7^{b}$ & $2.9^{b}$ & $3.2^{b, c}$ \\
\hline
\end{tabular}

${ }^{a}$ Calculated as the number of patients with MGUS divided by the number who were tested

$b$ Prevalence was age-adjusted to the 2000 US total population as follows: men, 4.0\% (95\% CI, 3.5-4.4); women, $2.7 \%$ (95\% CI, 2.4-3.0); total, $3.2 \%$ (95\% CI, 3.0-3.5)

${ }^{c}$ Prevalence was age-adjusted and sex-adjusted to the 2000 US total population

MGUS monoclonal gammopathy of undetermined significance (Adapted from Kyle et al. [7]) 